\title{
DNA Structural Changes Induced by Intermolecular Triple Helix Formation
}

\author{
Ibrahim Sayoh, ${ }^{\dagger} \S$ David A. Rusling, ${ }^{\dagger}$ Tom Brown, ${ }^{\ddagger 0}$ and Keith R. Fox* ${ }^{* \dagger}$ \\ ${ }^{\dagger}$ School of Biological Sciences, Life Sciences Building 85, University of Southampton, Southampton SO17 1BJ, U.K. \\ ${ }^{\ddagger}$ Department of Chemistry, University of Oxford, Oxford OX1 3TA, U.K.
}

\section{Supporting Information}

ABSTRACT: DNase I footprints of intermolecular DNA triplexes are often accompanied by enhanced cleavage at the $3^{\prime}$-end of the target site at the triplex-duplex junction. We have systematically studied the sequence dependence of this effect by examining oligonucleotide binding to sites flanked by each base in turn. For complexes with a terminal T.AT triplet, the greatest enhancement is seen with $\mathrm{ApC}$, followed by ApG and ApT, with the weakest enhancement at ApA. Similar DNase I enhancements were observed for a triplex with a terminal

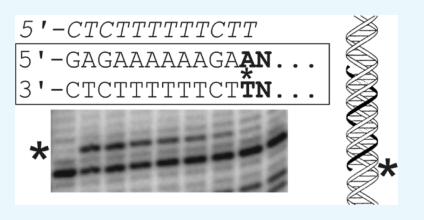
$\mathrm{C}^{+}$.GC triplet, though with little difference between the different GpN sites. Enhanced reactivity to diethylpyrocarbonate was observed at As that flank the triplex-duplex junction at $\underline{A} A \mathrm{~A}$ or $\underline{\mathrm{A} A C}$ but not $\underline{\mathrm{A} A G}$ or $\underline{\mathrm{A} A T}$. Fluorescence melting experiments demonstrated that the flanking base affected the stability with a $4{ }^{\circ} \mathrm{C}$ difference in $T_{\mathrm{m}}$ between a flanking $\mathrm{C}$ and $\mathrm{G}$. Sequences that produced the strongest enhancement correlated with those having the lower thermal stability. These results are interpreted in terms of oligonucleotide-induced changes in DNA structure and/or flexibility.

\section{INTRODUCTION}

Triplex-forming oligonucleotides (TFOs) bind sequenceselectively within the major groove of duplex DNA, forming specific hydrogen bonds to exposed groups on the base pairs (mainly to purines). ${ }^{1-4}$ Two main families of intermolecular triplexes have been described in which the third strand is either parallel or antiparallel to the purine-rich stand of the target. Parallel triplexes are characterized by T.AT and $\mathrm{C}^{+}$.GC triplets, ${ }^{4,5}$ while antiparallel complexes contain A.AT, G.GC, and T.AT triplets. ${ }^{6}$ Parallel triplexes usually require conditions of low $\mathrm{pH}(<6.0)$, which is necessary for protonation of $\mathrm{N} 3$ of the third strand cytosine. ${ }^{7,8}$ They also require the presence of divalent cations (magnesium), which is especially necessary for stabilizing the T.AT triplet. $^{9-11}$

Several biophysical techniques have been used to probe the affinity, selectivity, and structural effects of TFOs. Several highresolution NMR studies ${ }^{12-18}$ and a limited number of X-ray crystallographic studies ${ }^{19-21}$ have suggested that the underlying DNA duplex adopts a structure that is more like A-DNA, while retaining several B-like characteristics. UV and fluorescence melting studies have been used to determine the factors that affect triplex stability and to demonstrate selectivity for their intended target sequences, ${ }^{22,23}$ while DNase I footprinting studies have shown the location of TFO target sites on long DNA fragments and have been used to assess their selectivity and affinity. ${ }^{24,25}$ DNase I has been the most commonly used footprinting probe, although the oligopurine TFO target sites are often relatively poor substrates for this enzyme.

Because DNase I cuts from the DNA minor groove, while the TFO is positioned in the major groove, TFO footprints cannot be caused by direct steric blockage of the enzyme. DNase I cleavage efficiency is known to be affected by local
DNA structural variations, and $\mathrm{A}_{n} \cdot \mathrm{T}_{n}$ sequences are typically poor substrates for the enzyme on account of their narrow minor groove and rigid structure. ${ }^{26,27}$ Crystal structures of DNase I bound to short oligonucleotides showed that the DNA is bent away from the protein, and this distortion may be an essential part of the catalytic mechanism, explaining why sequences, such as $\mathrm{G}_{n} . \mathrm{C}_{n}$ are also poor substrates. ${ }^{28,29}$ TFOinduced DNase I footprints could therefore be due to TFOinduced DNA structural changes or variations in the duplex flexibility.

Several studies have noted that there is often enhanced DNase I cleavage at the 3 '-end of the TFO binding site at the triplex-duplex junction, and this is usually seen on the purinerich strand. ${ }^{30-35}$ Enhancements in reactivity to diethylpyrocarbonate (DEPC) have also been noted at this location. ${ }^{36}$ Similarly, enhanced sensitivity to cleavage by copperphenanthroline has been observed at the $3^{\prime}$-end of the purine strand at the triplex-duplex junction, ${ }^{37}$ which is also a strong binding site for ellipticine. ${ }^{38}$ These enhancements are restricted to the triplex-duplex junction and are only seen at a single bond at the $3^{\prime}$-end of the target and so cannot be explained by global changes in the ratio of the enzyme to free DNA. $^{39}$ The exposed base at the terminus of the third strand could create a site for stacking of agents such as phenanthroline and ellipticine, though this would not explain why these enhancements are only seen at the $3^{\prime}$ (not $5^{\prime}$ ) junction. Instead, these enhancements are thought to arise from TFOinduced changes in the local DNA structure that makes the phosphodiester bond at the duplex-triplex junction more

Received: November 6, 2019

Accepted: December 20, 2019

Published: January 15, 2020 
susceptible to DNase I cleavage. These enhancements are often more pronounced than the footprints themselves and so are sometimes evident in places where there is no clear DNase I footprint. ${ }^{40}$ In other instances, they have been used to estimate the location of the bound third strand. ${ }^{31}$

We have previously examined how the stability of the underlying duplex affects the apparent stability of the triplex, ${ }^{41}$ but to date, there have been no studies that systematically assess the sequence dependence of these TFO-induced enhancements. We have often used the tyr T DNA fragment for these footprinting experiments, which we later modified to include a 17 base oligopurine tract tyr $\mathrm{T}(43-59) .{ }^{42}$ The $3^{\prime}$-end of this tract ends in an ApC step and in the present study, we have changed this to ApX and GpX, where $\mathrm{X}=$ each base in turn and examined the interaction of these targets with 12-mer and 11-mer TFOs using DNase I footprinting as well as the reaction with DEPC. These studies have been augmented by fluorescence melting experiments with short oligonucleotides that are based on the same sequences.

\section{RESULTS}

Terminal T.AT Triplet. DNase I footprints for the interaction of the 12-mer-T oligonucleotide with four DNA fragments that contain the same triplex target site, but in which the $3^{\prime}-\mathrm{A}$ is followed by each base in turn, are shown in Figure 1. In each case, clear concentration-dependent footprints are

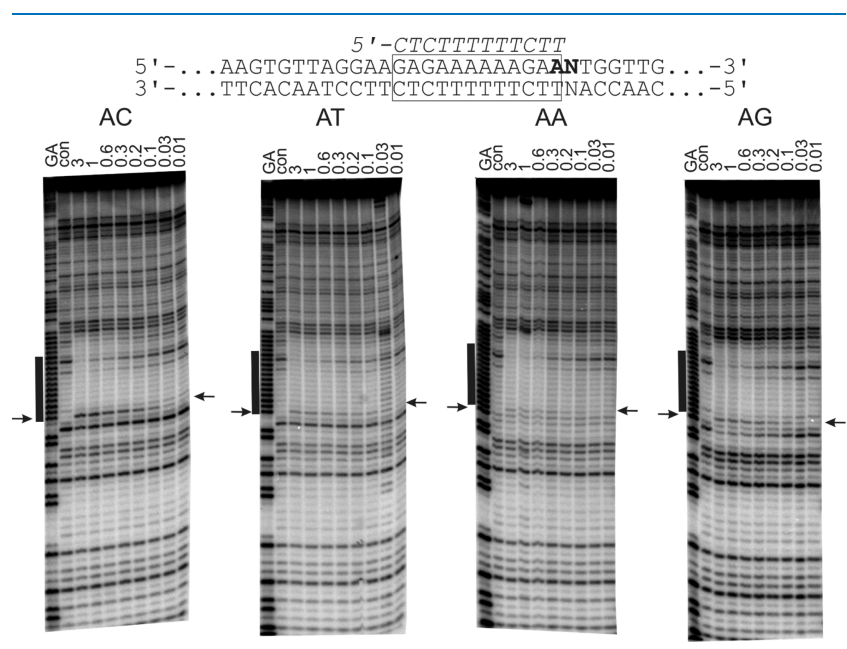

Figure 1. DNase I footprinting of the 12-mer-T oligonucleotide with fragments AX. The experiments were performed in $50 \mathrm{mM}$ sodium acetate $\mathrm{pH} 5.0$, containing $1 \mathrm{mM} \mathrm{MgCl}_{2}$. Oligonucleotide concentrations $(\mu \mathrm{M})$ are shown at the top of each gel lane. Con indicates the control in the absence of the added oligonucleotide and tracts labeled GA corresponding to markers for purines. The solid bars indicate the location of the target site for the 12-mer, while the arrows indicate the location of the enhancements at the $3^{\prime}$-end of the target site. The DNA was labeled at the $3^{\prime}$-end, so the gel runs $5^{\prime}-3^{\prime}$ from top to bottom.

evident that extend to TFO concentrations below $1 \mu \mathrm{M}$. In the original tyr $\mathrm{T}(43-59)$ sequence, the $3^{\prime}$-flanking base is $\mathrm{C}$, and results for this are shown in the left hand panel. As previously noted, the footprint extends above $\left(5^{\prime}-\right)$ the target site by about four nucleotides and is accompanied by enhanced cleavage at the $3^{\prime}$-(lower) triplex-duplex junction (indicated by the arrow). This enhancement shows similar concentration dependence to the footprint itself, and both are evident at concentrations around $1 \mu \mathrm{M}$. These enhancements have previously been interpreted as resulting from a triplex-induced conformational change at the triplex-duplex junction. Similar concentration-dependent enhancements are evident with the other fragments with T, A, and G flanking the $3^{\prime}-\mathrm{A}$. Visual inspection of these gels suggests that the enhancement is most pronounced for AC and weakest for AA. This might indicate that the dinucleotide $\mathrm{ApC}$ is most easily distorted by flanking triplex formation into a form that is most easily cleaved by DNase I.

Similar experiments for the interaction of the 11-mer-TFO with these sequences are shown in Figure 2A. The 11-mer TFO lacks the $3^{\prime}$-terminal nucleotide of 12 -mer- $\mathrm{T}$, and the base flanking the $3^{\prime}$-end of its target site is an A for all four fragments. The variable base is therefore one residue removed from the target site (i.e., AAN). DNase I footprints can be seen with all four fragments, though these require higher concentrations than with the 12 -mer TFO, as a result of its

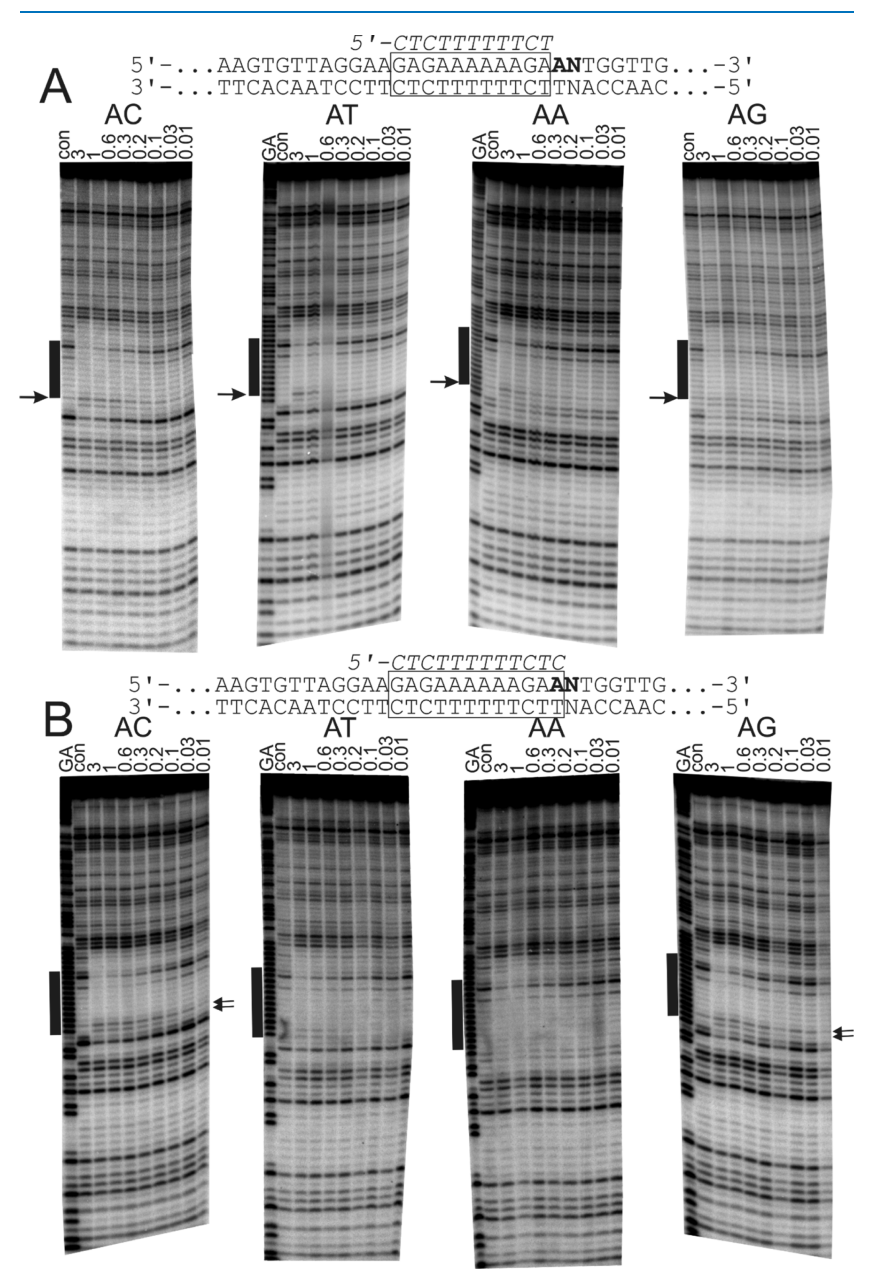

Figure 2. DNase I footprinting of oligonucleotides 11-mer (A) and 12 -mer-C (B) with fragments AX. The experiments were performed in $50 \mathrm{mM}$ sodium acetate $\mathrm{pH} 5.0$, containing $1 \mathrm{mM} \mathrm{MgCl}_{2}$. Oligonucleotide concentrations $(\mu \mathrm{M})$ are shown at the top of each gel lane. Con indicates the control in the absence of added oligonucleotide and tracts labeled GA correspond to markers for purines. The solid bars indicate the location of the 11-mer (A) and 12-mer (B) target sites. Note that 12 -mer-C generates a mismatched C.AT triplet at the lower $\left(3^{\prime}\right.$-end) of the target site. The arrows indicate the location of the enhancements at the $3^{\prime}$-end of the target site. The DNA was labeled at the $3^{\prime}$-end, so the gel runs $5^{\prime}-3^{\prime}$ from top to bottom. 
shorter length, and are only clearest at the highest concentrations $(3 \mu \mathrm{M})$. Enhanced DNase I cleavage (indicated by the arrows) is again evident at each of the triplex-duplex junctions, and as expected, this is one base higher than with the 12-mer-T TFOs. These enhancements are generally weaker than those seen with the 12-mer, and the intensity of the enhanced band is most pronounced with sequence $\mathrm{AC}$ and weakest with AA.

Similar experiments were performed with the 12-mer-C TFO, which differs from 12 -mer- $\mathrm{T}$ by replacing the $3^{\prime}-\mathrm{T}$ with $\mathrm{C}$, generating an 11-mer triplex of T.AT and C.GC triplets that is followed by a $3^{\prime}$-terminal C.AT triplet mismatch. Previous studies $^{31}$ suggested that some triplexes, such as this one, with terminal mismatches produce enhanced DNase I cleavage after both the canonical (T.AT) and noncanonical, mismatched (C.AT) triplets. The results of these experiments are shown in Figure $2 \mathrm{~B}$ and show similar concentration-dependent footprints to those seen with the 11-mer. In this instance, no enhancements are evident with the sequences AA and AT. In contrast, two weakly enhanced bands are seen with sequences $\mathrm{AC}$ and AG (indicated by the arrows). The upper of these bands is at the same position as seen with the 11-mer-TFO, corresponding to the triplex-duplex junctions after the canonical T.AT, while the bottom corresponds to the location of the mismatched C.AT triplet. Once again, the sequence AC produces the strongest enhancement of DNase I cleavage.

Terminal $\mathrm{C}^{+}$.GC Triplet. The results presented above describe triplexes that contain a $3^{\prime}$-terminal T.AT triplet (or a C.AT mismatch). In order to assess whether the identity of the terminal triplet affects these properties, we changed the base at the 3 '-end of the target oligopurine tract from A to G, generating four fragments in which this base $(G)$ is flanked by each base in turn (fragments GA, GC, GT, and GG). These form a 12-mer triplex with oligo 12 -mer-C that is similar to that with 12 -mer-T but ends in a $\mathrm{C}^{+}$.GC triplet instead of T.AT. DNase I footprinting experiments with these four new variants of the tyr T sequence are shown in Figure 3. Concentration-dependent footprints are evident with all four fragments, which persist to concentrations of about $0.2 \mu \mathrm{M}$. This is lower than the triplexes with the terminal T.AT triplet, as a result of the greater stability of the $\mathrm{C}^{+}$.GC triplet. All these footprints are accompanied by enhanced cleavage at the $3^{\prime}$-end of the target site, at the triplex-duplex junction. In this instance, the intensity of the enhanced bands is similar for all four flanking sequences.

We also investigated the interaction of the 11-mer TFO with these target sites that end in a $3^{\prime}-\mathrm{G}$. This triplex is identical to that formed between this TFO and the targets ending in A, though the immediate $3^{\prime}$-flanking base is G instead of A (AGN instead of AAN). The results of these DNase I footprinting experiments are shown in Figure 4A and show concentrationdependent footprints that persist to a concentration of about 1 $\mu \mathrm{M}$. This is about 10 times higher than the concentration that is required to produce DNase I footprints with 12 -mer-C at this target sequence and is similar to that seen with the 11-merTFO and the AN sequences. These footprints are again accompanied by enhanced cleavage, which as expected, is located one band higher than with 12-mer-C. The intensities of these enhancements are similar for all four sequences and are stronger than those with the AN targets, even though the underlying triplex is identical.

We also examined the interaction of the 12 -mer-T TFO with the target sites that contain a 3 '-guanine. This should generate

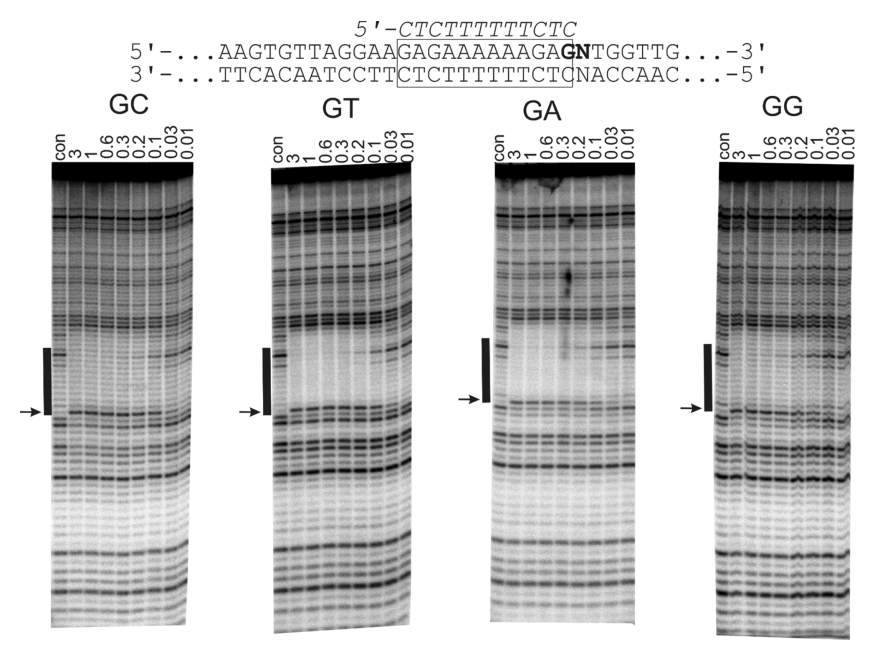

Figure 3. DNase I footprinting of the 12-mer-C oligonucleotide with fragments GX. The experiments were performed in $50 \mathrm{mM}$ sodium acetate $\mathrm{pH} 5.0$, containing $1 \mathrm{mM} \mathrm{MgCl}_{2}$. Oligonucleotide concentrations $(\mu \mathrm{M})$ are shown at the top of each gel lane. Con indicates the control in the absence of the added oligonucleotide. The solid bars indicate the location of the target site for the oligonucleotide, while the arrows indicate the location of the enhancements at the $3^{\prime}$-end of the target site. The DNA was labeled at the $3^{\prime}$-end, so the gel runs $5^{\prime}-3^{\prime}$ from top to bottom.

a triplex with 11 canonical triplets $\left(\mathrm{C}^{+}\right.$.GC and T.AT) ending with T.AT, followed by a mismatched T.GC triplet. DNase I footprints for this interaction are shown in Figure 4B. This produces DNase I footprints that are only apparent at the highest TFO concentrations $(3 \mu \mathrm{M})$. These footprints are accompanied by only weak enhancements, which are one base higher than those seen with 12 -mer- $\mathrm{C}$, at the same position as seen with the 11-mer, with no enhancements at the mismatched terminal T.GC triplet.

Reaction with DEPC. DEPC mainly reacts at N7 of adenines. Its reaction is generally poor in duplex DNA, but it has been used to detect unusual or distorted DNA structures in which this base is more exposed. ${ }^{43,44}$ Enhanced reactivity has previously been demonstrated at a triplex-duplex junction. ${ }^{36}$ Figure 5 shows the results of DEPC cleavage experiments with the four AN fragments, in the presence the TFOs 12-mer-T, 11-mer, and 12-mer-C. The control lanes of these footprints show some reaction with As within the oligopurine tract (especially in the run of six consecutive As) and at the $3^{\prime}$-end of the oligopurine tracts of AA and AT (but not AG and AC). DEPC cleavage at these sites is attenuated by interaction with the TFOs in a concentration-dependent manner, as expected, as the TFO binds to N7 of A and prevents access to the probe. There is no evidence of any ligand-induced enhanced DEPC reactivity in the presence of 12 -mer-T oligonucleotide (Figure $5 \mathrm{~A}$; first four panels), even with sequence $\mathrm{AA}$, which contains an A immediately adjacent to the TFO binding site.

Similar experiments with the 11-mer TFO are shown in the middle four panels of this figure (Figure $5 \mathrm{~B}$ ). In contrast to the results with 12 -mer- $T$, enhanced reaction to DEPC is seen at the $3^{\prime}$-end of the TFO binding site in the sequence AA, and to a lesser extent in $\mathrm{AC}$, though no enhancements are apparent with AT and AG. These enhancements are located at the A that is immediately $3^{\prime}$ - to the 11-mer binding site (i.e., at AAC and $\mathrm{AAA}$, with sequences $\mathrm{AC}$ and $\mathrm{AA}$, respectively). 


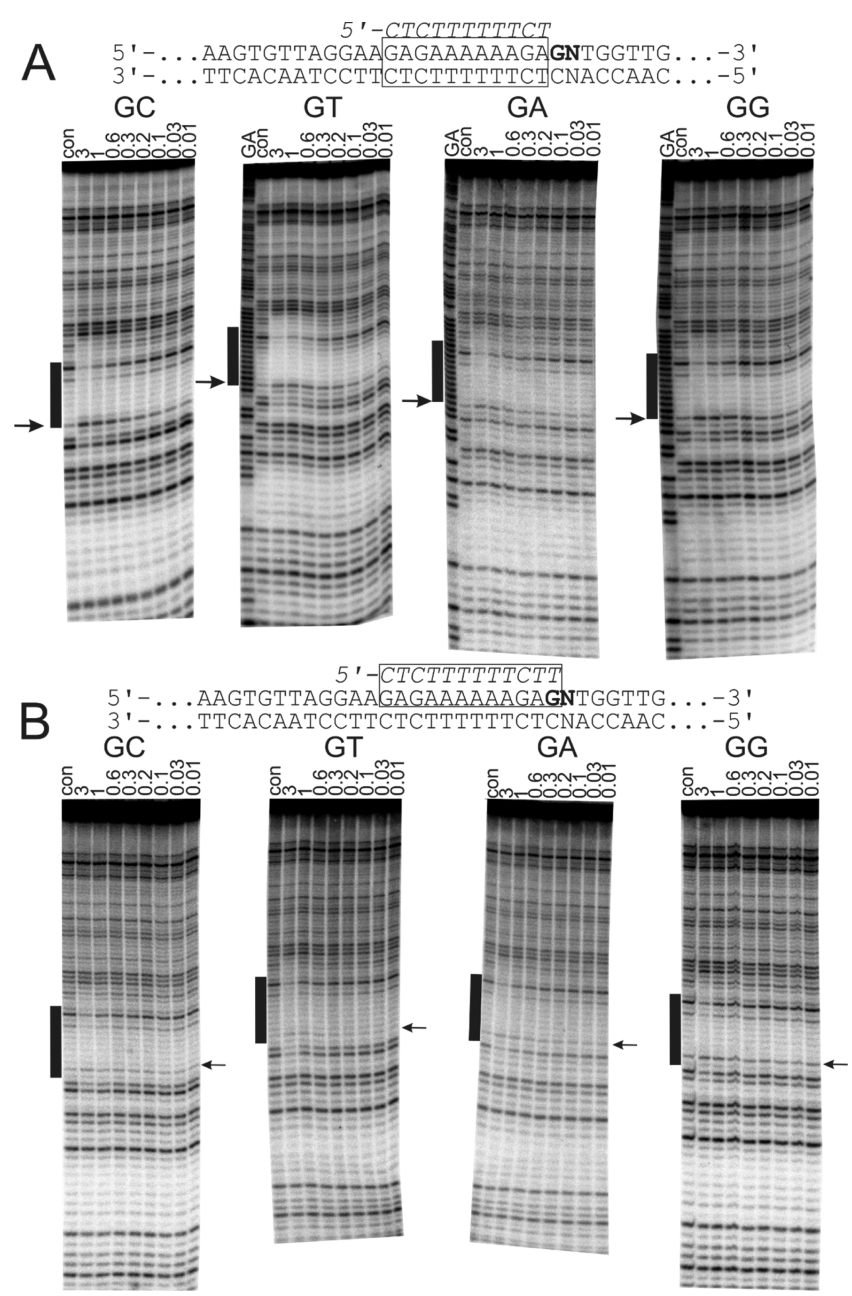

Figure 4. DNase I footprinting of oligonucleotides 11-mer (A) and 12-mer-T (B) with fragments GX. The experiments were performed in $50 \mathrm{mM}$ sodium acetate $\mathrm{pH} 5.0$, containing $1 \mathrm{mM} \mathrm{MgCl}_{2}$. Oligonucleotide concentrations $(\mu \mathrm{M})$ are shown at the top of each gel lane. Con indicates the control in the absence of added oligonucleotide, and tracts labeled GA correspond to markers for purines. The solid bars indicate the location of the 11-mer (A) and 12-mer (B) target sites. Note that 12-mer-T generates a mismatched T.GC triplet at the lower $\left(3^{\prime}\right.$-end) of the target site. The arrows indicate the location of the enhancements at the $3^{\prime}$-end of the target site. The DNA was labeled at the $3^{\prime}$-end, so the gel runs $5^{\prime}-3^{\prime}$ from top to bottom.

Similar experiments showing the reaction with DEPC in the presence of 12-mer-C shown are in the final four panels of Figure 5 (Figure 5C). These cleavage patterns are similar to those formed with the 11-mer TFO and again show enhanced DEPC reactivity at the $3^{\prime}$-end of the triplex-duplex junction in the sequences $\mathrm{AC}$ and $\mathrm{AA}$ (at $\mathrm{A} \underline{\mathrm{AC}}$ and $\mathrm{A} \underline{\mathrm{A} A}$, respectively), and is again especially strong for AA.

Other Probes. We also examined the effect of these TFOs on the reaction with permanganate (reacting with exposed Ts), micrococcal nuclease (cleaving at pA and pT), and hydroxyl radicals (generating an even ladder of cleavage products). None of the TFO target combinations induced any enhanced reaction with any of these probes.

Triplex Stability. The experiments described above demonstrate that triplex formation can affect the susceptibility of flanking bases to some enzymes and chemical cleavage agents. However, these techniques are not sufficiently sensitive to detect any changes in triplex affinity. We therefore examined the stability of triplexes that are flanked by different base pairs by thermal melting studies using fluorescently labeled synthetic oligonucleotides. In these experiments, the 12-mer third strand TFO was labeled at the $5^{\prime}$-end with dabcyl, while the purine strand of the target duplex was labeled at its 5 -end with fluorescein. The sequences of these oligonucleotides are shown in Table 1 and were chosen to correspond to the 12-mer target site in the tyr T fragments. When the triplex is assembled, the fluorophore and quencher are in close proximity and the fluorescence is quenched. The triplex melts when the temperature is increased, separating the fluorophore and quencher, leading to a large increase in fluorescence. These experiments were performed in $50 \mathrm{mM}$ sodium acetate $\mathrm{pH} 5.0$ containing $1 \mathrm{mM} \mathrm{MgCl} 2$ and $200 \mathrm{mM} \mathrm{NaCl}$.

Fluorescence melting curves for the four target duplexes in the presence of $5 \mu \mathrm{M}$ of the 12-mer-T oligonucleotide are presented in Figure 6A, and $T_{\mathrm{m}}$ values derived from these are presented in Table 2 . These results show a clear difference in stability of these different complexes, even though they all contain the same triplex. There is a $4{ }^{\circ} \mathrm{C}$ difference in $T_{\mathrm{m}}$ between the highest (AG) and lowest (AC).

The results of similar experiments with the 11-mer TFO on these four target sequences are shown in Figure $6 \mathrm{~B}$ and Table 2. As expected, these 11-mer triplexes melt at lower temperatures than those of the 12-mer- $T$ triplexes, but again, we find that the different complexes display significantly different melting temperatures. These differences are less pronounced than for the 12-mer triplexes but the triplex with sequence $\mathrm{AC}$ melts about $2{ }^{\circ} \mathrm{C}$ lower than $\mathrm{AG}$, even though these sequences only differ at two base pairs distal to the triplex target site.

We also examined the stability of similar triplexes that contain a terminal $3^{\prime}-\mathrm{C}^{+}$.GC triplet instead of T.AT, using target duplexes that contain a GC base pair at the $3^{\prime}$-end of the polypurine tract, flanked by each base in turn. The melting curves of these four targets with TFO-12-mer- $\mathrm{C}$ are shown in Figure $6 \mathrm{C}$, and the $T_{\mathrm{m}} s$ are presented in Table 2. Sequence GT has the highest $T_{\mathrm{m}}\left(49.7{ }^{\circ} \mathrm{C}\right)$, though this is only slightly higher than GC $\left(49.3{ }^{\circ} \mathrm{C}\right)$, GA $\left(48.7^{\circ} \mathrm{C}\right)$, and GG $\left(48.6^{\circ} \mathrm{C}\right)$. As expected, these $T_{\mathrm{m}}$ values are higher than those formed with the four targets with a $3^{\prime}$-terminal T.AT triplet as a result of the greater stability of the $\mathrm{C}^{+}$.GC triplet.

The results of similar experiments with the shorter 11-mer TFO on these four target sequences flanked by a GC base pair are also shown in Table 2. As expected, the $T_{\mathrm{m}} \mathrm{s}$ of these 11mer triplexes are between 8 and $10{ }^{\circ} \mathrm{C}$ lower than those of their 12-mer counterparts. This is a smaller reduction than between the 11-mer and 12-mer-T for the targets ending with an AT base pair, reflecting the greater additional stability that is afforded by the $\mathrm{C}^{+}$.GC triplet compared to T.AT. As expected, there are only small differences between the $T_{\mathrm{m}} s$ of these 11 -mer complexes, which only vary by a single base pair that is located two base pairs distal to the triplex.

Figure $6 \mathrm{D}$ shows the results of melting experiments with the $\mathrm{AN}$ target duplexes and the 12-mer-C TFO, generating a C.AT mismatch at the $3^{\prime}$-end of the triplex. The $T_{\mathrm{m}}$ values estimated from these data are shown in Table 2. Although these triplexes have slightly different melting temperatures, the differences are much less pronounced than with the 12-mer-T. Similar experiments were also performed with the 12 -mer- $T$ and the GN-targets sequences, generating a T.GC mismatch at the $3^{\prime}$ end of the triplex and the $T_{\mathrm{m}} s$ are shown in Table 2 . It can be 


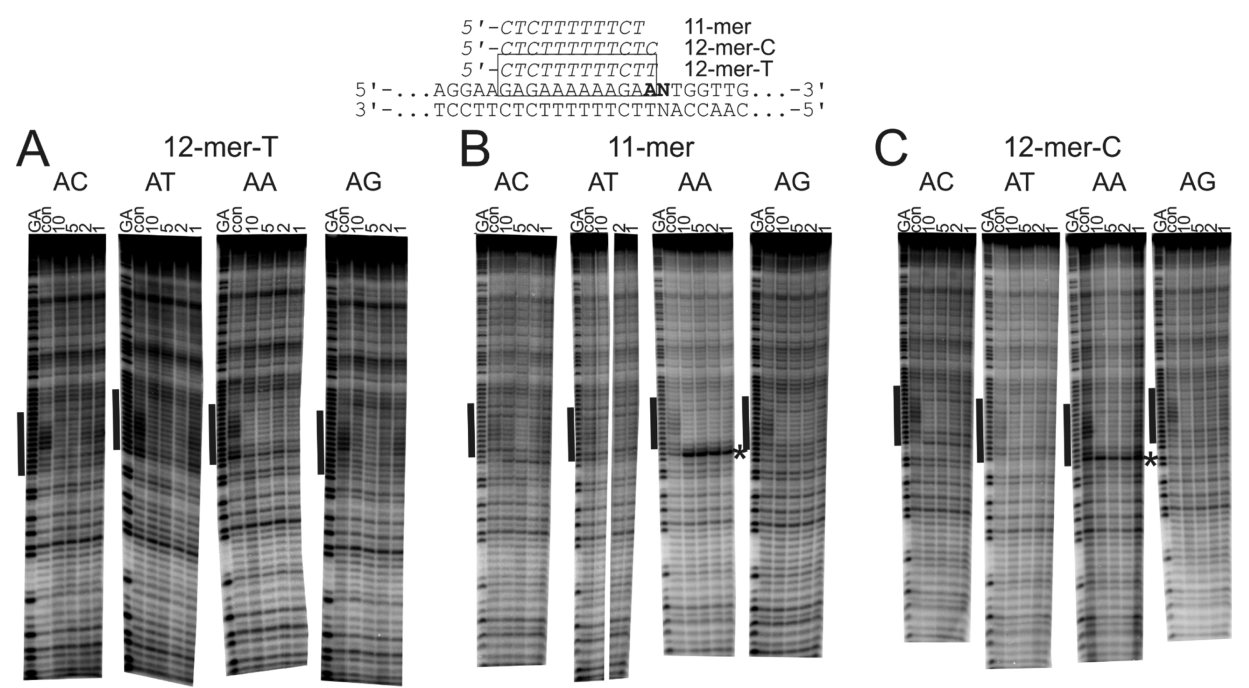

Figure 5. DEPC footprinting of oligonucleotides 12-mer-T (A), 11-mer (B), and 12-mer-C with fragments AX. The experiments were performed in $50 \mathrm{mM}$ sodium acetate $\mathrm{pH} 5.0$, containing $1 \mathrm{mM} \mathrm{MgCl}$. Oligonucleotide concentrations $(\mu \mathrm{M})$ are shown at the top of each gel lane. Con indicates the control in the absence of the added oligonucleotide and tracts labeled GA correspond to markers for purines. The solid bars indicate the location of the target sites for 12-mer-T (A), 11-mer (B), and 12-mer-C (C). Note that 12-mer-C generates a mismatched C.AT triplet at the lower ( $3^{\prime}$-end) of the target site. The asterisks indicate the location of the enhancements at the $3^{\prime}$-end of the target site. The DNA was labeled at the $3^{\prime}-$ end, so the gel runs $5^{\prime}-3^{\prime}$ from top to bottom.

Table 1. Oligonucleotides Used in Fluorescence Melting Experiments $^{a}$

$\begin{array}{lc} & \text { dabcyl-labeled TFOs } \\ \text { 12-mer-T } & 5^{\prime}-\mathrm{Q} \text {-СTCTTTTTTCTT-3' } \\ \text { 12-mer-C } & 5^{\prime}-\mathrm{Q} \text {-СTCTTTTTTCTC-3' } \\ \text { 11-mer } & 5^{\prime} \text {-Q-CTCTTTTTTCT-3' }\end{array}$

\begin{tabular}{|c|c|}
\hline Targ & uplexes used to form 12-mer triplexes w \\
\hline$A C$ & $\begin{array}{r}5^{\prime}-\mathrm{F}-\text { GAGAAAAAAGAACTGGTTG-3' } \\
3^{\prime} \text {-CTCTTTTTTCTTGACCAAC-5' }\end{array}$ \\
\hline AG & 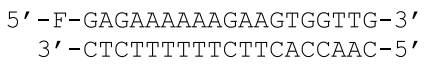 \\
\hline $\mathrm{AA}$ & $\begin{array}{r}5^{\prime}-\mathrm{F}-\text { GAGAAAAAAGAAACGGTTG-3' } \\
3^{\prime} \text {-CTCTTTTTTCTTTGCCAAC-5' }\end{array}$ \\
\hline AT & $\begin{array}{l}5^{\prime}-\mathrm{F} \text {-GAGAAAAAAGAATTGGTTG-3' } \\
3^{\prime} \text {-CTCTTTTTTCTTAACCAAC-5' }\end{array}$ \\
\hline
\end{tabular}

Target duplexes used to form 12-mer triplexes with a $3^{\prime}$-C+.GC triplet

GC 5'-F-GAGAAAAAAGAGCTGGTTG-3'

$3^{\prime}$-СTCTTTTTTCTCGACCAAC-5'

GG 5'-F-GAGAAAAAAGAGGTGGTTG-3'

3'-СТСТTТTTTСТССАССАAС-5'

GA 5'-F-GAGAAAAAAGAGATGGTTG-3'

$3^{\prime}$-СТСТTTTTTCTCTACCAAC-5'

GT 5'-F-GAGAAAAAAGAGTTGGTTG-3'

3'-CTCTTTTTTCTCAACCAAC-5'

${ }^{a} \mathrm{Q}=$ dabcyl, $\mathrm{F}=$ fluorescein. The pyrimidine-containing TFOs were labeled with $5^{\prime}$-dabcyl, while the target duplexes were labeled with fluorescein at the $5^{\prime}$-end of the purine strands. The variant nucleotides at the $3^{\prime}$-end of the target are shown in bold.

seen that there are no significant differences between these melting curves. Comparing the $T_{\mathrm{m}}$ of these triplexes with those generated by the fully matched 12 -mer, it can be seen that the terminal mismatch decreases the triplex stability by about 10 ${ }^{\circ} \mathrm{C}$ (Table 2). It appears that addition of a $3^{\prime}$-T.GC is destabilizing compared to the 11-mer, while addition of a $3^{\prime}$ C.AT triplet increases triplex stability.

\section{DISCUSSION}

These results demonstrate that a common feature of intermolecular DNA triplex formation is the presence of enhanced DNase I cleavage at the triplex-duplex junction at the 3 '-end of the oligopurine strand of the target. For triplexes with a terminal T.AT triplet, the strongest enhancement is seen when this is followed by an ApC step (sequence AC), with the weakest observed at ApA. In principle, it would be best to compare cleavage of this band in the triplex, with that in the uncomplexed DNA, in order to assess the fold enhancement in the presence of the TFO at each target site. However, this is often not possible to determine, as cleavage of this region is vanishingly low in the absence of the oligonucleotide. However, we have estimated the relative enhancements at these steps by comparing the intensity of the enhanced band with cleavage of other regions of the fragments that are not affected by triplex binding. These results are shown in Table 3 and confirm the strongest enhancement at ApC followed by ApG and ApT, with the weakest at ApA.

We assume that these enhancements reflect triplex-induced changes in the local DNA structure. How might these be reflected in altered DNase I activity? Crystal structures of DNase I bound to short oligonucleotides reveal that the enzyme functions by inserting an exposed loop into the DNA minor groove. Regions with a narrow groove (such as $\mathrm{A}_{n} \cdot \mathrm{T}_{n}$ tracts) are therefore poor substrates for the enzyme. Ligandinduced changes in groove width have previously been used to explain enhancements adjacent to some small-molecule binding sites. ${ }^{45,46}$ However, these changes are unlikely to account for these TFO-induced enhancements, as they are restricted to a single phosphodiester bond at the triplexduplex junction. A circular permutation assay demonstrated that triplex formation is accompanied by DNA stiffening, rather than a junctional bending model, an effect that may also explain why third strand binding in the major groove inhibits DNase cleavage from the minor groove. ${ }^{47}$ We can envisage two other possibilities to account for these changes. The BI to BII phosphate backbone configuration is known to depend on the 

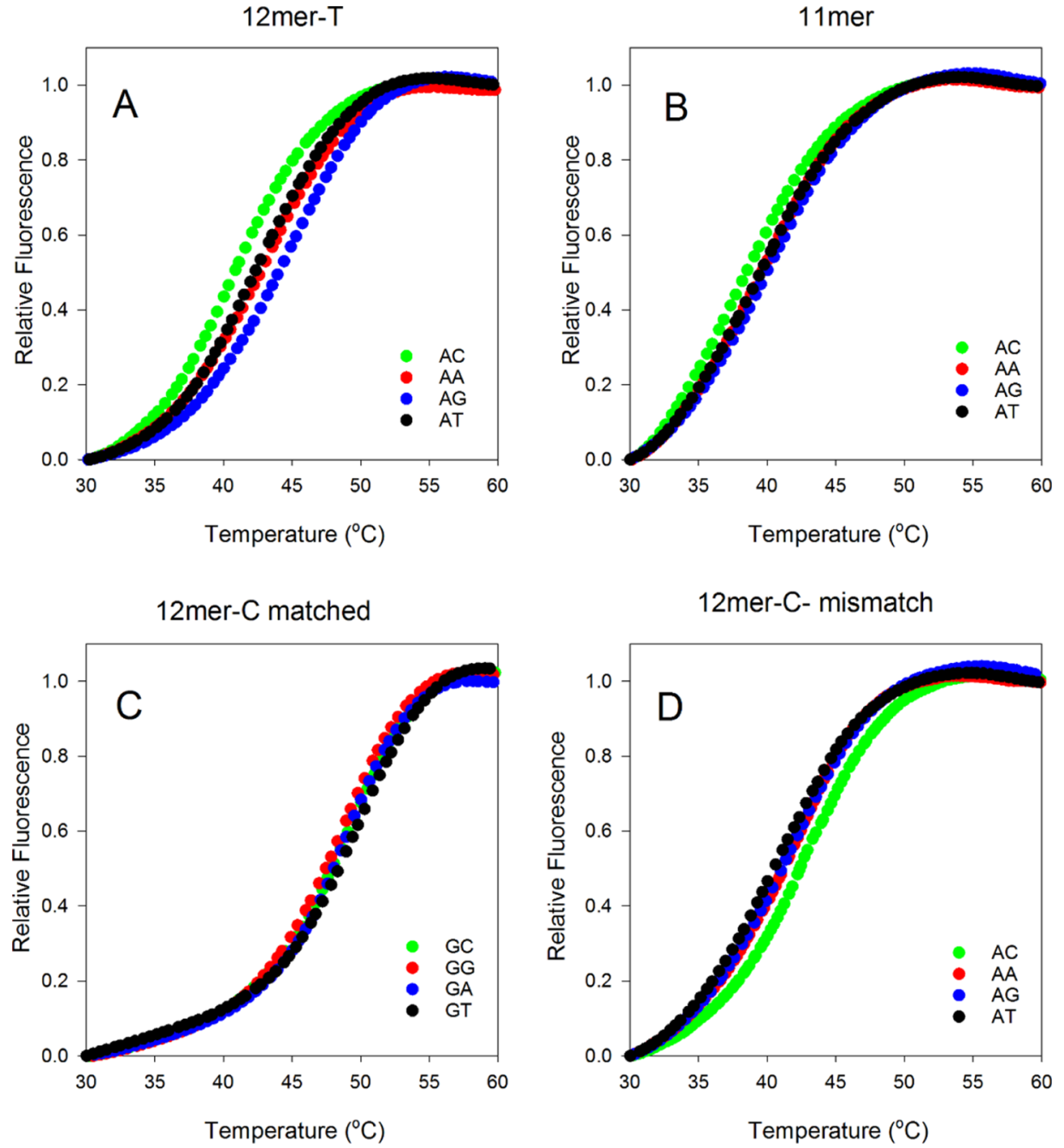

Figure 6. Fluorescence melting curves for the interaction of $5 \mu \mathrm{M}$ dabcyl-labeled 12-mer-T, 11-mer, and 12-mer-C TFOs with the target sites AX and GX. Experiments were performed in $50 \mathrm{mM}$ sodium acetate $\mathrm{pH} 5.0$, containing $200 \mathrm{mM} \mathrm{NaCl}$ and $1 \mathrm{mM} \mathrm{MgCl}$. The duplex purine strand was labeled at the $5^{\prime}$-end with fluorescein. The target duplex concentration was $0.25 \mu \mathrm{M}$. (A) Interaction of 12 -mer-T with AX, (B) interaction of 11mer with $\mathrm{AX},(\mathrm{C})$ interaction of 12-mer $\mathrm{C}$ with GX, and (D) interaction of 12-mer-C with $\mathrm{AX}$.

Table 2. $T_{\mathrm{m}}$ Values $\left({ }^{\circ} \mathrm{C}\right)$ for the Interaction of the Three TFOs with Different Target Sites ${ }^{a}$

$\begin{array}{cccc}\text { sequence } & 12 \text {-mer-T } & 11 \text {-mer } & \text { 12-mer-C } \\ \text { AC } & 40.7 \pm 0.4 & 38.2 \pm 0.5 & 42.8 \pm 0.2 \\ \text { AG } & 45.0 \pm 0.3 & 40.2 \pm 0.2 & 41.8 \pm 0.2 \\ \text { AA } & 43.7 \pm 0.3 & 40.4 \pm 0.2 & 42.1 \pm 0.4 \\ \text { AT } & 42.3 \pm 0.2 & 39.8 \pm 0.3 & 40.7 \pm 0.2 \\ \text { GC } & 36.4 \pm 0.2 & 39.7 \pm 0.4 & 49.3 \pm 0.1 \\ \text { GG } & 37.6 \pm 0.3 & 40.4 \pm 0.3 & 48.6 \pm 0.1 \\ \text { GA } & 38.5 \pm 0.2 & 40.9 \pm 0.2 & 48.7 \pm 0.1 \\ \text { GT } & 38.4 \pm 0.4 & 41.0 \pm 0.1 & 49.7 \pm 0.1\end{array}$

${ }^{a}$ Reactions were performed in $50 \mathrm{mM}$ sodium acetate $\mathrm{pH} 5.0$ containing $200 \mathrm{mM} \mathrm{NaCl}$. The target duplex concentration was 0.2 $\mu \mathrm{M}$ with $5 \mu \mathrm{M}$ TFO.

local dinucleotide sequence, and DNase I favors the BII configuration. $^{48}$ It may be that triplex formation favors the BII configuration at the triple-duplex boundary, thereby enhancing enzyme cleavage. An alternative, though related, explanation is that DNase I is known to bend the DNA away from the minor groove at the cleavage site, and this bending may be an important part of the enzyme's catalytic mechanism. ${ }^{28,29,49}$
Table 3. Relative Intensities of the Enhanced Bands at the Highest Concentration of Oligonucleotide 12-mer-T (3 $\mu \mathrm{M})$, Relative to Bands in the Rest of the Fragment That are Not Affected by the Oligonucleotide ${ }^{a}$

$\begin{array}{cc}\text { target sequence } & \text { relative enhancement (arbitrary units) } \\ \text { AC } & 1.00 \pm 0.01 \\ \text { AG } & 0.54 \pm 0.04 \\ \text { AA } & 0.38 \pm 0.01 \\ \text { AT } & 0.62 \pm 0.05\end{array}$

${ }^{a_{T}}$ The data were derived from gels shown in Figure 1.

Triplex formation may therefore alter the local deformability of the scissile phosphodiester bond. This is consistent with the observation that $\mathrm{ApC}$ is known to be one of the most deformable dinucleotides and ApA is one of the most rigid. ${ }^{50,51}$ Such changes in DNA flexibility may not be restricted to the immediate sequence, but can be propagated into neighboring sequences, and thereby account for the observation that the 11-mer produced greater enhancement with AC than the other three dinucleotides, even though the ApC step was one base removed from the enhancement. Although it is clear that triplex formation generates enhanced cleavage at the triple- 
duplex junction, the precise origin of this effect will probably require comparison of crystal structures of a duplex in the presence and absence of a TFO.

These enhancements are not restricted to flanking ApN sites, but are also evident at $\mathrm{GpN}$, at triplex-duplex junctions, following a $3^{\prime}$-terminal $\mathrm{C}^{+}$.GC triplet. However, these enhancements are less sequence-dependent and are similar magnitude for all $\mathrm{GpN}$ sites. It may be that the presence of a $\mathrm{C}^{+}$.GC triplet at the end of the triplex (instead of T.AT), which is known to give greater affinity, makes it more rigid and less deformable.

The fluorescence melting experiments with oligonucleotides containing different flanking sequences showed subtle but significant changes in triplex stability, especially for the ApN series, with a $4{ }^{\circ} \mathrm{C}$ difference between the highest (ApG) and the lowest $(\mathrm{ApC})$. It may be significant that the sequences with the lowest $T_{\mathrm{m}}$ generally correspond to the ones that produce the greatest enhancement in DNase I cleavage. It may be that, for the less stable complexes, some of the TFO binding energy is used to distort the DNA helix, or affect its dynamic flexibility, thereby reducing the inherent stability of the complex. In light of this, the targeting of oligopurine sites by TFOs might be improved by considering the flexibility of this flanking sequence.

The changes in reactivity to DEPC were surprising and showed no changes for interaction of the 12-mer $\mathrm{T}$ with any of the sequences, even at $\mathrm{AA}$, with an $\mathrm{A}$ as the $3^{\prime}$-adjacent base. In contrast, the shorter (11-mer) sequence produced clear enhancements with the sequence $\mathrm{AA}$ and also with $\mathrm{AC}$, that is, in the sequences $\underline{A A A}$ and $\underline{A} A C$ (in which the underlined base corresponds to the terminal triplet and the one in bold shows enhanced DEPC reactivity). This result is similar to that with the 12 -mer $\mathrm{C}$ at these sequences (producing 11 canonical triplets and ending with a C.AT mismatch), for which DEPC enhancements were again observed with $\mathrm{AA}$ and $\mathrm{AC}$. It therefore seems that DEPC enhancements are generated in sequences $\mathrm{AA}(\mathrm{A} / \mathrm{C})$ but not $\mathrm{A} A(\mathrm{G} / \mathrm{T})$ (the interaction of the 12-mer $\mathrm{T}$ with $\mathrm{AA}$ ends with the sequence AAT).

These results confirm that enhanced DNase I cleavage is a common feature at the 3 '-end of triplex-duplex junctions. The concentration dependence of these enhancements is similar to that of the footprints themselves and has previously been useful for confirming triplex formation at target sequences with very poor DNase I cleavage. ${ }^{31,40}$ In general, the strongest enhancements are seen with complexes that have lower thermal stability. The effect of flanking bases on triplex stability and structure may therefore need to be considered when optimizing triplex target design.

\section{EXPERIMENTAL SECTION}

Oligonucleotides. Three TFOs were used for the footprinting experiments: $5^{\prime}$-CTCTTTTTTCTT (12-mer-T), 5'-CTCTTTTTTCTC (12-mer-C), and 5'-CTCTTTTTTCT (11-mer). These were provided by ATDBio and were synthesized using an Applied Biosystems ABI 394 automated DNA/RNA synthesizer using solid-phase DNA phosphoramidite synthesis cycles. For the fluorescence melting experiments, similar TFOs were prepared with $5^{\prime}$-dabcyl (Q) on the TFO and $5^{\prime}$-fluorescein on the duplex purine strand. The oligonucleotides were purified by gel filtration, dissolved in water, and kept at $-20{ }^{\circ} \mathrm{C}$ until required. Oligonucleotides for site-directed mutagenesis were obtained from ATDBio and are listed in Table S1.
DNA Sequences for Footprinting. The base at the 3 'end of the oligopurine tract in tyr $\mathrm{T}(43-59)$ is an $\mathrm{A}$, followed by $\mathrm{C}$ presenting an $\mathrm{ApC}$ step at the triplex-duplex boundary. The $\mathrm{C}$ at position 42 was changed to each base in turn by QuickChange PCR using the pairs of primers, as shown in Table S1. A set of four other derivatives was also prepared in which the A at position 43 was changed to $G$, followed by each of the other bases in turn, using the primers which are also shown in Table S1. The resulting plasmids were transformed into competent Escherichia coli TG2 cells, and plasmids were prepared using a Qiagen Miniprep kit. The sequences of the resulting plasmids were confirmed by MWG Eurofins. In this work, the eight fragments are designated by the bases at positions 43 and 42 (i.e., AC, AG, AT, AA, GC, GG, GT, and GA).

Plasmids containing the oligopurine target sites were digested with EcoRI and AvaI and labeled at the $3^{\prime}$-end of the EcoRI site with $\alpha-{ }^{32} \mathrm{P}$ dATP using either reverse transcriptase or exo-Klenow fragment. The fragments of interest were separated from the remainder of the plasmid DNA on $6 \%$ polyacrylamide gels, eluted, and dissolved in 10 $\mathrm{mM}$ Tris- $\mathrm{HCl} \mathrm{pH} 7.4$ containing $0.1 \mathrm{mM}$ ethylenediaminetetraacetic acid (EDTA) at a concentration of about $10 \mathrm{cps} / \mu \mathrm{L}$, as determined on a hand-held Geiger Counter.

DNase I Footprinting. TFO $(3 \mu \mathrm{L})$ diluted in $50 \mathrm{mM}$ sodium acetate $\mathrm{pH} 5.0$ containing $1 \mathrm{mM} \mathrm{MgCl} 2$ was mixed with $1.5 \mu \mathrm{L}$ of radiolabeled DNA and incubated at room temperature for $2 \mathrm{~h}$. DNase I cleavage was performed by adding $2 \mu \mathrm{L}$ of DNase I (diluted to about 0.1 units $/ \mathrm{mL}$ in 20 $\mathrm{mM} \mathrm{NaCl}, 2 \mathrm{mM} \mathrm{MgCl}$ and $2 \mathrm{mM} \mathrm{MnCl}_{2}$ ) and digested for 2 $\min$. The reactions were stopped by adding $5 \mu \mathrm{L}$ of DNase I stop solution containing $80 \%$ formamide, $10 \mathrm{mM}$ EDTA, and $0.01 \%$ bromophenol blue. The samples were heated at $100{ }^{\circ} \mathrm{C}$ for $3 \mathrm{~min}$ and crash-cooled on ice before subjecting to denaturing polyacrylamide gel electrophoresis.

Reaction with DEPC. TFO $(3 \mu \mathrm{L})$ diluted in $50 \mathrm{mM}$ sodium acetate $\mathrm{pH} 5.0$ containing $1 \mathrm{mM} \mathrm{MgCl}$ was mixed with $1.5 \mu \mathrm{L}$ of radiolabeled DNA and incubated at room temperature for $2 \mathrm{~h}, 3 \mu \mathrm{L}$ of DEPC was then added, and the reaction was left for $30 \mathrm{~min}$ with occasional mixing. The reaction was stopped by adding $2 \mu \mathrm{L}$ of $3 \mathrm{M}$ sodium acetate, and the DEPC-modified products were precipitated, cleaved by adding $50 \mu \mathrm{L}$ of $10 \%(\mathrm{v} / \mathrm{v})$ piperidine, heated at $100{ }^{\circ} \mathrm{C}$ for 30 $\mathrm{min}$ and dried in a vacuum centrifuge. The pellets were washed with water, dried again, and dissolved in $8 \mu \mathrm{L}$ of DNase I stop solution.

Gel Electrophoresis. The products of DNase I or DEPC digestion were separated on $8 \%$ denaturing polyacrylamide gels containing $8 \mathrm{M}$ urea. Gels were run at $1500 \mathrm{~V}$ for about $90 \mathrm{~min}$ and then fixed in $10 \%$ acetic acid and dried onto Whatman 3 MM paper. Dried gels were exposed to phosphor screens overnight which were scanned with a Typhoon phosphorimager.

Fluorescence Melting. Fluorescence melting experiments were performed as previously described. ${ }^{23}$ For these experiments, the TFOs were labeled with 5-dabcyl (Q), that is, $5^{\prime}-\mathbf{Q}$ CTСТTTTTTCTT, 5'-Q-CTCTTTTTTCTC, and 5'-QСТСТТТтТТСТ. The target duplexes were labeled with fluorescein at the $5^{\prime}$-end of the purine strand, that is, $5^{\prime}$-FGAGAAAAAAGARXTGGTTG, where $\mathrm{R}=\mathrm{A}$ or $\mathrm{G}$ and $\mathrm{X}=$ each base in turn; these were annealed with the complementary oligonucleotides 5'-CAACCAXYTCTTTTTTCTC 
( $\mathrm{Y}=\mathrm{C}$ or $\mathrm{T}$ and $\mathrm{X}=$ each base in turn). These sequences are listed in Table 1.

Melting profiles were determined using a Roche LightCycler in a total volume of $20 \mu \mathrm{L}$ in $50 \mathrm{mM}$ sodium acetate $\mathrm{pH} 5.0$, containing $200 \mathrm{mM} \mathrm{NaCl}$ and $1 \mathrm{mM} \mathrm{MgCl}_{2}$. The duplex concentration was $0.25 \mu \mathrm{M}$ for all experiments, with TFO concentrations of $9,5,3,1$, and $0.25 \mu \mathrm{M}$. The mixtures were annealed by heating to $98^{\circ} \mathrm{C}$ for $5 \mathrm{~min}$ and cooling to $35^{\circ} \mathrm{C}$ at $0.1{ }^{\circ} \mathrm{C} / \mathrm{s}$; the reaction was held at $35{ }^{\circ} \mathrm{C}$ for $5 \mathrm{~min}$ before heating to $98{ }^{\circ} \mathrm{C}$ at $0.1{ }^{\circ} \mathrm{C} / \mathrm{s}$. The fluorescence profile was recorded for both the annealing and melting phases, and no hysteresis was observed for any of these sequences. Melting temperatures $\left(T_{\mathrm{m}}\right)$ were estimated from the maxima in the first derivatives of the melting profiles using the LightCycler software.

\section{ASSOCIATED CONTENT}

\section{S Supporting Information}

The Supporting Information is available free of charge at https://pubs.acs.org/doi/10.1021/acsomega.9b03776.

Sequence of the oligonucleotides used in QuickChange PCR to generate the eight triplex targets $\mathrm{AN}$ and GN, where $\mathrm{N}$ is each base in turn (PDF)

\section{AUTHOR INFORMATION}

\section{Corresponding Author}

*E-mail: k.r.fox@soton.ac.uk.

\section{ORCID $\odot$}

Tom Brown: 0000-0002-6538-3036

Keith R. Fox: 0000-0002-2925-7315

\section{Present Address}

${ }^{\S}$ Faculty of Science and Technology, Princess of Naradhiwas University, Narathiwat, Thailand 96000.

\section{Notes}

The authors declare no competing financial interest.

\section{REFERENCES}

(1) Felsenfeld, G.; Davies, D. R.; Rich, A. Formation of a threestranded polynucleotide molecule. J. Am. Chem. Soc. 1957, 79, 20232024.

(2) Thuong, N. T.; Hélène, C. Sequence-specific recognition and modification of double-helical DNA by oligonucleotides. Angew. Chem., Int. Ed. Engl. 1993, 32, 666-690.

(3) Fox, K. Targeting DNA with triplexes. Curr. Med. Chem. 2000, 7, 17-37.

(4) Moser, H.; Dervan, P. Sequence-specific cleavage of double helical DNA by triple helix formation. Science 1987, 238, 645-650.

(5) Doan, T. L.; Perrouault, L.; Praseuth, D.; Habhoub, N.; Decout, J. L.; Thuong, N. T.; Lhomme, J.; Héène, C. Sequence-specific recognition, photo-cross-linking and cleavage of the DNA double helix by an oligo- $[\alpha]$-thymidylate covalently linked to an azidoproflavine derivative. Nucleic Acids Res. 1987, 15, 7749-7760.

(6) Beal, P.; Dervan, P. Second structural motif for recognition of DNA by oligonucleotide-directed triple-helix formation. Science 1991, 251, 1360-1363.

(7) Sugimoto, N.; Wu, P.; Hara, H.; Kawamoto, Y. pH and cation effects on the properties of parallel pyrimidine motif DNA triplexes. Biochemistry 2001, 40, 9396-9405.

(8) Collier, D. A.; Wells, R. D. Effect of length, supercoiling, and $\mathrm{pH}$ on intramolecular triplex formation. Multiple conformers at pur.pyr mirror repeats. J. Biol. Chem 1990, 265, 10652-10658.

(9) James, P. L.; Brown, T.; Fox, K. R. Thermodynamic and kinetic stability of intermolecular triple helices containing different proportions of $\mathrm{C}^{+}$.GC and T.AT triplets. Nucleic Acids Res. 2003, $31,5598-5606$.

(10) Floris, R.; Scaggiante, B.; Manzini, G.; Quadrifoglio, F.; Xodo, L. E. Effect of cations on purine purine pyrimidine triple helix formation in mixed-valence salt solutions. Eur. J. Biochem. 1999, 260, 801-809.

(11) Malkov, V. A.; Voloshin, O. N.; Soyfer, V. N.; FrankKamenetskii, M. D. Cation and sequence effects on stability of intermolecular pyrimidine-purine-purine triplex. Nucleic Acids Res. 1993, 21, 585-591.

(12) Asensio, J. L.; Lane, A. N.; Dhesi, J.; Bergqvist, S.; Brown, T. The contribution of cytosine protonation to the stability of parallel DNA triple helices. J. Mol. Biol. 1998, 275, 811-822.

(13) Asensio, J. L.; Brown, T.; Lane, A. N. Solution conformation of a parallel DNA triple helix with 5' and 3' triplex-duplex junctions. Structure 1999, 7, 1-11.

(14) De los Santos, C.; Rosen, M.; Patel, D. NMR studies of DNA $(\mathrm{R}+)_{\mathrm{n}} \cdot(\mathrm{Y}-)_{\mathrm{n}} \cdot(\mathrm{Y}+)_{\mathrm{n}}$ triple helices in solution: Imino and amino proton markers of $\mathrm{T} \cdot \mathrm{A} \cdot \mathrm{T}$ and $\mathrm{C} \cdot \mathrm{G} \cdot \mathrm{C}^{+}$base-triple formation. Biochemistry $1989,28,7282-7289$.

(15) Radhakrishnan, I.; Gao, X.; De los Santos, C.; Live, D.; Patel, D. J. NMR Structural Studies of Intramolecular $(\mathrm{Y}+)_{\mathrm{n}} \cdot(\mathrm{R}+)_{\mathrm{n}}(\mathrm{Y}-)_{\mathrm{n}}$ DNA Triplexes in Solution - Imino and Amino Proton and Nitrogen Markers of G.TA Base Triple Formation. Biochemistry 1991, 30, 9022-9030.

(16) Radhakrishnan, I.; Patel, D. J. Solution/structure of an intramolecular purine-purine-pyrimidine DNA triplex. J. Am. Chem. Soc. 1993, 115, 1615-1617.

(17) Radhakrishnan, I.; Patel, D. J. Solution Structure and Hydration Patterns of a Pyrimidine?Purine?Pyrimidine DNA Triplex Containing a Novel T?CG Base-triple. J. Mol. Biol. 1994, 241, 600-619.

(18) Radhakrishnan, I.; Patel, D. J. DNA triplexes: Solution structures, hydration sites, energetics, interactions, and function. Biochemistry 1994, 33, 11405-11416.

(19) Rhee, S.; Han, Z.-j.; Liu, K.; Miles, H. T.; Davies, D. R. Structure of a triple helical DNA with a triplex-duplex junction. Biochemistry 1999, 38, 16810-16815.

(20) Abdallah, H. O.; Ohayon, Y. P.; Chandrasekaran, A. R.; Sha, R.; Fox, K. R.; Brown, T.; Rusling, D. A.; Mao, C.; Seeman, N. C. Stabilisation of self-assembled DNA crystals by triplex-directed photocross-linking. Chem. Commun. 2016, 52, 8014-8017.

(21) Van Meervelt, L.; Vlieghe, D.; Dautant, A.; Gallois, B.; Précigoux, G.; Kennard, O. High-resolution structure of a DNA helix forming (C.G)*G base triplets. Nature 1995, 374, 742-744.

(22) Mergny, J.-L.; Lacroix, L. Analysis of thermal melting curves. Oligonucleotides 2003, 13, 515-537.

(23) Darby, R. A. J.; Sollogoub, M.; McKeen, C.; Brown, L.; Risitano, A.; Brown, N.; Barton, C.; Brown, T.; Fox, K. R. High throughput measurement of duplex, triplex and quadruplex melting curves using molecular beacons and a LightCycler. Nucleic Acids Res. 2002, 30, No. e39.

(24) Hampshire, A.; Rusling, D.; Broughtonhead, V.; Fox, K. Footprinting: A method for determining the sequence selectivity, affinity and kinetics of DNA-binding ligands. Methods 2007, 42, 128140.

(25) Rusling, D. A.; Powers, V. E. C.; Ranasinghe, R. T.; Wang, Y.; Osborne, S. D.; Brown, T.; Fox, K. R. Four base recognition by triplex-forming oligonucleotides at physiological pH. Nucleic Acids Res. 2005, 33, 3025-3032.

(26) Drew, H. R.; Travers, A. A. DNA structural variations in the Escherichia-coli tyrT-promoter. Cell 1984, 37, 491-502.

(27) Drew, H. R. Structural specificities of five commonly used DNA nucleases. J. Mol. Biol. 1984, 176, 535-557.

(28) Suck, D.; Lahm, A.; Oefner, C. Structure refined to $2 \AA$ of a nicked DNA octanucleotide complex with DNase-I. Nature 1988, 332, 464-468.

(29) Lahm, A.; Suck, D. DNase I-induced DNA conformation. $2 \AA$ Structure of a DNase I-octamer complex. J. Mol. Biol. 1991, 222, 645-667. 
(30) Bijapur, J.; Bergqvist, S.; Brown, T.; Keppler, M. D.; Fox, K. R. 5-(1-propargylamino)-2 '-deoxyuridine $\left(\mathrm{U}^{\mathrm{P}}\right)$ : a novel thymidine analogue for generating DNA triplexes with increased stability. Nucleic Acids Res. 1999, 27, 1802-1809.

(31) Cardew, A. S.; Brown, T.; Fox, K. R. Secondary binding sites for heavily modified triplex forming oligonucleotides. Nucleic Acids Res. 2012, 40, 3753-3762.

(32) Cassidy, S. A.; Strekowski, L.; Wilson, W. D.; Fox, K. R. Effect of a triplex-binding ligand on parallel and antiparallel DNA triple helices using short unmodified and acridine-linked oligonucleotides. Biochemistry 1994, 33, 15338-15347.

(33) Fox, K. R. Formation of DNA Triple Helices Incorporating Blocks of G.GC and T.AT Triplets Using Short Acridine-Linked Oligonucleotides. Nucleic Acids Res. 1994, 22, 2016-2021.

(34) Sollogoub, M.; Darby, R. A. J.; Cuenoud, B.; Brown, T.; Fox, K. R. Stable DNA triple helix formation using oligonucleotides containing 2'-aminoethoxy,5-propargylamino-U. Biochemistry 2002, $41,7224-7231$.

(35) Stonehouse, T. J.; Fox, K. R. DNase-I footprinting of triplehelix formation at polypurine tracts by acridine-linked oligopyrimidines - Stringency, structural-changes and interaction with minorgroove binding ligands. Biochim. Biophys. Acta, Gene Struct. Expression 1994, 1218, 322-330.

(36) Collier, D. A.; Mergny, J.-L.; Thuong, N. T.; Helene, C. SiteSpecific Intercalation at the Triplex-Duplex Junction Induces A Conformational Change Which Is Detectable by Hypersensitivity to Diethylpyrocarbonate. Nucleic Acids Res. 1991, 19, 4219-4224.

(37) François, J.-C.; Saison-Behmoaras, T.; Hélène, C. SequenceSpecific Recognition of the Major Groove of DNA by Oligodeoxynucleotides Via Triple Helix Formation - Footprinting Studies. Nucleic Acids Res. 1988, 16, 11431-11440.

(38) Perrouault, L.; Asseline, U.; Rivalle, C.; Thuong, N. T.; Bisagni, E.; Giovannangeli, C.; Doan, T. L.; Hélène, C. Sequence-specific artificial photo-induced endonucleases based on triple helix-forming oligonucleotides. Nature 1990, 344, 358-360.

(39) Goodisman, J.; Dabrowiak, J. C. Structural changes and enhancements in DNase I footprinting experiments. Biochemistry 1992, 31, 1058-1064.

(40) Chandler, S. P.; Fox, K. R. Triple-Helix Formation at $\mathrm{A}_{8} \mathrm{XA}_{8} \cdot \mathrm{T}_{8} \mathrm{YT}_{8}$. FEBS Lett. 1993, 332, 189-192.

(41) Rusling, D. A.; Rachwal, P. A.; Brown, T.; Fox, K. R. The stability of triplex DNA is affected by the stability of the underlying duplex. Biophys. Chem. 2009, 145, 105-110.

(42) Brown, P. M.; Madden, C. A.; Fox, K. R. Triple-helix formation at different positions on nucleosomal DNA. Biochemistry 1998, 37, 16139-16151.

(43) Jeppesen, C.; Nielsen, P. E. Detection of intercalation-induced changes in DNA-structure by reaction with diethyl pyrocarbonate or potassium-permanganate - evidence against the induction of Hoogsteen base-pairing by echinomycin. FEBS Lett. 1988, 231, 172-176.

(44) Kahl, B. F.; Paule, M. R. The Use of Diethyl Pyrocarbonate and Potassium Permanganate as Probes for Strand Separation and Structural Distortions in DNA. In DNA-Protein Interactions: Principles and Protocols, 3rd ed.; Moss, T., LeBlanc, B., Eds.; Springer, 2009; pp 73-85.

(45) Fox, K. R.; Waring, M. J. DNA structural variations produced by actinomycin and distamycin as revealed by DNAase-I footprinting. Nucleic Acids Res. 1984, 12, 9271-9285.

(46) Low, C. M. L.; Drew, H. R.; Waring, M. J. Sequence-specific binding of echinomycin to DNA - evidence for conformationalchanges affecting flanking sequences. Nucleic Acids Res. 1984, 12, $4865-4879$.

(47) Maher, L. J.; Dervan, P. B.; Wold, B. Analysis of promoterspecific repression by triple-helical DNA complexes in a eukaryotic cell-free transcription system. Biochemistry 1992, 31, 70-81.

(48) Heddi, B.; Abi-Ghanem, J.; Lavigne, M.; Hartmann, B. Sequence-Dependent DNA Flexibility Mediates DNase I Cleavage. J. Mol. Biol. 2010, 395, 123-133.
(49) Suck, D.; Oefner, C. Structure of DNase-I at $2.0 \AA$ A resolution suggests a mechanism for binding to and cutting DNA. Nature 1986, $321,620-625$.

(50) Travers, A. A. The structural basis of DNA flexibility. Philos. Trans. R. Soc., A 2004, 362, 1423-1438.

(51) El Hassan, M. A.; Calladine, C. R. Propeller-twisting of basepairs and the conformational mobility of dinucleotide steps in DNA. J. Mol. Biol. 1996, 259, 95-103. 